\title{
Education Symposium
}

\author{
Published online: 6 May 2021
}

\section{E-1}

Navigating the Next Generation Sequencing Transcriptomics Landscape. RANCE NAULT. Department of Biochemistry \& Molecular Biology, Institute for Integrative Toxicology, Michigan State University, 1129 Farm Ln., East Lansing, MI 48824. Email: naultran@msu.edu

RNA-Seq has become a mainstay of biological research. From experimental design to data analysis, possessing a good understanding of the fundamentals is essential to producing high quality data and interpreting findings. In this workshop we will take a high-level look at the landscape of next generation sequencing transcriptomic approaches and technologies. Topics will cover key considerations for all stages of an RNA-Seq experiment from experimental design to library preparation strategies, sequencing parameter selection, and computational tools for read alignment and downstream analyses. Emerging single-cell RNA-sequencing technology will be introduced and contrasted to traditional bulk RNA-seq highlighting their advantages and disadvantages. This workshop is aimed at preparing students for their first bulk or single-cell RNA-sequencing experiment by highlighting key considerations when evaluating the diverse technologies and approaches in this continuously expanding area of research.

\section{E-2}

What Every Biologist Needs to Know About Artificial Intelligence. L. D. BURGOON. Raptor Pharm \& Tox, Ltd., 501 W Williams St \#1911, Apex, NC 27502. Email: burgoon.lyle@raptorpharmtox.com

Artificial Intelligence - the almighty buzzword. Depending upon who you read, and what you want to do, it can either solve your problems, or create new ones - in truth, it does both. In this talk I will cut through the hype and attempt to break down artificial intelligence, and specifically machine learning (the most abundant form in the wild), to the bare nuts and bolts. I will discuss the different types of machine learning algorithms that in vitro biologists are the most likely to run into, and give the highlights of the pros and cons. Machine learning at the end of the day is not different from much of what we do in science, and I will draw the parallels between the concepts of "garbage-in/garbage-out", the importance of data quality, the importance of study design, and the difference between causality and association. I will discuss the importance of validation in machine learning, and how machine learning models should be validated (versus how they typically are), and why this all matters. I will also briefly talk about symbolic artificial intelligence, a branch of artificial intelligence that I also work in, but that is largely eclipsed by machine learning. By the end of the talk, the audience should be able to quickly vet machine learning models they encounter in the wild (e.g., scholarly papers, news reports), and be able to identify what methods might work best to make predictions they care about. 